\title{
THE TWO-SIDED EXIT PROBLEM FOR SPECTRALLY POSITIVE LÉVY PROCESSES
}

\author{
L. C. G. ROGERS, ${ }^{*}$ University of Cambridge
}

Let $\left(X_{t}\right)_{t>0}$ be a (real-valued) Lévy process, let $\bar{X}_{t} \equiv \sup _{s \leqq t} X_{s}$, let $P^{x}$ denote the law of $X$ started at $x \in \mathbb{R}$, and let $H_{x} \equiv \inf \left\{t>0: X_{t}=x\right\}$ be the first-hitting time of $x$. To avoid trivialities, we shall assume that neither $X$ nor $-X$ is a subordinator.

If $\tau_{a b} \equiv \inf \left\{t>0: X_{t} \notin(a, b)\right\}$, where $a<0<b$, the two-sided exit problem is the problem of identifying the law of the pair $\left(\tau_{a b}, X\left(\tau_{a b}\right)\right)$ under $P^{0}$. For a general Lévy process, there is no useful solution to this problem, and even if we assume (as we shall from now on) that the Lévy process is spectrally positive (that is, has no downward jumps), the formulae which can be obtained (see, for example, $\S 6$ of [1]) are still very complicated-with one exception. This one simple result admits a simple proof, which it is the purpose of this note to explain. The result, due in essence to Emery [3] and Takács [4] p. 37, is stated as follows.

Theorem. There exists a continuous increasing $F: \mathbb{R}^{+} \rightarrow \mathbb{R}^{+}$such that $0<x<y$

$$
h(x, y) \equiv P^{x}\left(H_{0}<\infty, \bar{X}\left(H_{0}\right) \leqq y\right)=F(y-x) / F(y) .
$$

In the case where $P^{0}\left(\sup _{t} X_{t}<\infty\right)=1, F$ may be taken to be the distribution function of $\bar{X}_{\infty} \equiv \sup _{t} X_{t}$, and in the case where $P^{0}\left(\sup _{t} X_{t}=\infty\right)=1, F$ may be taken to be

$$
F(x)=\lim _{N \rightarrow \infty} P^{0}\left(\bar{X}\left(H_{-N}\right) \leqq x \mid H_{-N}<\infty\right) e^{\alpha x},
$$

where $P^{0}\left(\right.$ inf $\left._{t} X_{t}<y\right)=e^{a y}$ for $y<0$.

Proof. Firstly, for $0<\varepsilon<y$ we have

$$
\begin{aligned}
h(\varepsilon, y) & =P^{0}\left[H_{-\varepsilon}<\infty, \bar{X}\left(H_{-\varepsilon}\right) \leqq y-\varepsilon\right] \\
& \rightarrow 1 \text { as } \varepsilon \downarrow 0,
\end{aligned}
$$

since by the Blumenthal $0-1$ law, $P^{0}\left(\inf \left\{t>0: X_{t}<0\right\}=0\right)=0$ or 1 , and the value 0 is impossible for a spectrally positive Lévy process unless $X$ is a subordinator, which was excluded.

Spectral positivity implies that if $X$ starts at $x>0$ and reaches 0 , then $X$ must pass through each $z \in(0, x)$ on its way to 0 . Hence, using the strong Markov property at $H_{z}$, we have easily

$$
h(x, y)=h(x-z, y-z) . h(z, y) \text { for } 0<z<x<y .
$$

Defining $g(a, b)$ for $0<b<a$ by $g(a, b) \equiv h(a-b, a)$, this is rephrased as

$$
g(a, b)=g(c, b) g(a, c) \text { for } 0<b<c<a .
$$

Hence for $0<b<c<a$,

$$
g(c, b)=\frac{g(a, b)}{g(a, c)}
$$

Received 23 January 1990.

* Postal address: Statistical Laboratory, Department of Pure Mathematics and Mathematical Statistics, University of Cambridge, 16 Mill Lane, Cambridge, CB2 1SB. 
Since the left-hand side does not depend on $a$, nor does the right-hand side, so we may unambiguously define a function $F$ by

From this immediately

$$
F(b)=\frac{g(a, b)}{g(a, 1)} \text { for } b>0, \quad a>b \vee 1
$$

which is rephrased as

$$
g(c, b)=F(b) / F(c) \text { for } 0<b<c,
$$

$$
h(x, y)=\frac{F(y-x)}{F(y)} \text { for } 0<x<y,
$$

establishing (1). Now observe that $h(x, y)$ is decreasing in $x$, from the definition of $h$, and right-continuous, by (3), (4). Hence $F$ is increasing and left-continuous. If $F$ had a discontinuity at $y_{0}$, say, then pick $x_{0} \in\left(0, y_{0}\right)$ such that $y_{0}-x_{0}$ is a continuity point of $F$. Then, from (6), $h\left(x_{0}, \cdot\right)$ jumps downward at $y_{0}$, in contradiction of the definition of $h$. Hence $F$ is continuous, and the first statement of the theorem is proved.

When $P\left(\sup _{t} X_{t}<\infty\right)=1$, we note that for $a>b$

$$
\begin{aligned}
g(a, b) & =P^{0}\left(H_{b-a}<\infty, \bar{X}\left(H_{b-a}\right) \leqq b\right) \\
& \rightarrow P^{0}\left(\bar{X}_{\infty} \leqq b\right) .
\end{aligned}
$$

When $P\left(\sup _{t} X_{t}=\infty\right)=1$, then by the strong Markov property, - inf $_{t} X_{t}$ has an exponential distribution with parameter $\alpha \geqq 0$, say; the parameter $\alpha$ is zero if $P\left(\inf _{t} X_{t}=-\infty\right)=1$, otherwise $\alpha$ is the unique positive real such that $\psi(-\alpha)=0$, where $\psi$ is the Lévy exponent of $X$ (see [1]). Then

$$
e^{\alpha(a-b)} g(a, b)=P^{0}\left(\bar{X}\left(H_{b-a}\right) \leqq b \mid H_{b-a}<\infty\right) .
$$

Rearrangement and letting $a \rightarrow \infty$ yields (2).

Remark. We observe in passing that, since $X$ can hit points, by Theorem 1 of [2] the resolvent kernel has a bounded density, providing a proof of Corollary 1 on p. 733 of [1].

\section{Acknowledgement}

It is a pleasure to thank Nick Bingham for suggesting I look at this result.

\section{References}

[1] Bingham, N. H. (1975) Fluctuation theory in continuous time. Adv. Appl. Prob. 7, 705-766.

[2] BRETAGnolle, J. (1971) Résultats de Kesten sur les processus à accroissements indépendants. Sém. de Probabilités V, Lecture Notes in Mathematics 191, Springer-Verlag, Berlin, 21-36.

[3] Emery, D. J. (1973) Exit problem for a spectrally positive process. Adv. Appl. Prob. 5, 498-520. York.

[4] TAKÁCs, L. (1967) Combinatorial Methods in the Theory of Stochastic Processes. Wiley, New 